\title{
Study on salvianolic acid $B$ in the reduction of epidural fibrosis in laminectomy rats
}

\author{
Feng Chen, Zhenbo Zuo, Kai Wang, Chengdong Zhang, Haifeng Gong, Fagang Ye, Aiyu Ji and Hao Tao*
}

\begin{abstract}
Background: Epidural fibrosis (EF) is a common complication after laminectomy. Salvianolic acid B (Sal B) is a major bioactive component of a traditional Chinese medical agent, Salvia miltiorrhiza, which has shown anti-inflammatory, anti-fibrotic and anti-proliferative properties. The object of this study was to investigate the effect of Sal B on the prevention of epidural fibrosis in laminectomy rats.

Methods: A controlled double-blinded study was conducted in sixty healthy adult Wistar rats that underwent laminectomy at the L1-L2 levels. The rats were randomly divided into 3 groups of 20: (1) Sal B treatment group; (2) Vehicle group; (3) Sham group (laminectomy without treatment). All rats were sacrificed 4 weeks post-operatively. The extent of epidural fibrosis, fibroblast proliferation and the expression of vascular endothelial growth factor (VEGF) and inflammatory factors were analyzed.
\end{abstract}

Results: The recovery of all rats was uneventful. In the laminectomy sites treated with Sal B, the dura mater showed no adhesion. Collagen deposition was significantly lower in the Sal B group than the other two groups. In addition, both fibroblast and inflammatory cell counting in the laminectomy sites treated with Sal B showed better grades than the other two groups. The expression of VEGF and inflammatory factors in operative sites also suggested better results in the Sal B group than the other two groups.

Conclusions: Sal B inhibits fibroblast proliferation, blood vessel regeneration, and inflammatory factor expression. Thus, Sal $B$ is able to prevent epidural scar adhesion in post-laminectomy rats.

Keywords: Epidural fibrosis, Salvianolic acid B, Laminectomy, Rat

\section{Background}

Failed back surgery syndrome (FBSS) is gaining attention from both lumbar surgeons and lumbar laminectomy patients. FBSS occurs in $8-40 \%$ of patients who undergo lumbar disc surgery [1]. Recurrent persistent low back pain and chronic nerve radicular are the main characteristics for FBSS [2]. Epidural fibrosis (EF) is widely accepted to be the main contributor to FBSS [3-5].

$\mathrm{EF}$, as a scar tissue adjacent to the dura mater following lumbar laminectomy, can lead to extensive nerve roots and dural mater adhesions. Therefore, EF could cause restriction of nerve root mobility, dural compression and spinal canal stenosis [6].

Various attempts have been made to prevent EF, such as topical application of immunosuppressive agents [7],

\footnotetext{
*Correspondence: haotao2015@126.com

Department of Trauma, Affiliated Hospital of Qingdao University, Qingdao, PR China
}

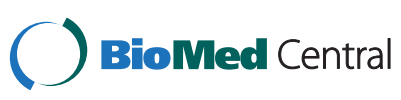

(c) 2014 Chen et al.; licensee BioMed Central Ltd. This is an Open Access article distributed under the terms of the Creative Commons Attribution License (http://creativecommons.org/licenses/by/4.0), which permits unrestricted use, distribution, and reproduction in any medium, provided the original work is properly credited. The Creative Commons Public Domain Dedication waiver (http://creativecommons.org/publicdomain/zero/1.0/) applies to the data made available in this article unless otherwise stated. anti-inflammatory agents [8], calcium channel blockers [6], animal collagen membranes [9], modified meticulous surgery [10], and traditional Chinese medical agents, such as pseudo-ginseng and Angelica sinensis [5,11]. Although some of them have achieved moderate success in animals, there is still no single agent or biomaterial that has reached the success of clinic application.

Salvianolic acid B (Sal B), a major bioactive component of the traditional Chinese medical agent, Salvia miltiorrhiza, is widely accepted for use against cardiovascular diseases [12]. Sal B is reported to exert anti-inflammatory and neuroprotective effects both in vivo and in vitro [13-15]. And with the advantages of little or no toxicity, Sal B is also able to alleviate liver fibrosis [16]. However, there have been no studies investigating the effects of Sal $\mathrm{B}$ on preventing EF.

In the present study in laminectomized rats, we evaluated the efficacy of Sal B in the prevention of EF. Specifically, 
macroscopic assessment, histological analysis, and measurement of inflammatory factors and hydroxyproline content were applied.

\section{Methods}

\section{Subjects}

A total of sixty adult healthy male Wistar rats (mean weight $250 \mathrm{~g}$ ) were employed. The present research was approved by Qingdao University Medical College Medical Ethics Committee. In compliance with the principles of International Laboratory Animal Care and with the European Communities Council Directive (86/809 /EEC), animals were housed in the local laboratory under the conditions of 20 to $25^{\circ} \mathrm{C}$ room temperature, a 12 hour light-dark cycle and clean food and water ad libitum (Additional file 1). All efforts were made to minimize animal numbers and suffering. The rats were housed for 10 days to adjust them to the environment pre-operatively. Animals were randomly divided into three groups (20 rats in each group): 1) Sal B treatment group (Sal B $30 \mathrm{mg} / \mathrm{kg}$, diluted in saline); 2) Vehicle group (saline); 3) Sham group (laminectomy without treatment).

\section{Agents and antibodies}

Sal B (purity $>98 \%$ ) was purchased from the National Institute for the Control of Pharmaceutical and Biological Products (Beijing, China). Cal-EX II solution for decalcification and dehydration was purchased from Thermo Fisher Scientific (Waltham, MA, USA). $\beta$ Dimethylaminobenzaldehyde was purchased from SigmaAldrich (St. Louis, MO, USA). Reverse Transcriptase was purchased from Promega (Madison MA, USA). Primary antibody and secondary antibodies were purchased from abcam (Cambridge, UK).

\section{Surgery}

The rat laminectomy model was created as previously reported [4-7]. The surgery was performed under sterile conditions with basic surgical tools. Anesthetization was performed by intra-peritoneal injection of $10 \%$ chloral hydrate $(0.3 \mathrm{ml} / 100 \mathrm{~g}$ body weight $)$. All the rats were numbered individually after being restrained on a warm pad in the prone position. The fur of each rat was shaved around L1 and L2 and the exposed skin was sterilized. After that L1-L2 total laminectomy was performed with a micro-rongeur. Close attention was paid not to traumatize the dura and the nerve roots. After complete hemostasis with saline, the wound site was surgically closed.

\section{Salvianolic acid B administration}

In the Sal B treatment group, salvianolic acid B $(30 \mathrm{mg} / \mathrm{kg}$, diluted in saline) was administered intragastrically [17]. In the Vehicle group, the same volume of saline was given in

\section{Table 1 Primer sequences}

\begin{tabular}{ll}
\hline TGF-ß1 (148 bp) & forward, 5'-GCCCTGCCCCTACATTGGG-3' \\
IL-6 (131 bp) & reverse, 5'-CTTGCGACCCACGTAGTAGACGAT-3'; \\
& reverse, 5'-TGCCGAGTAGACCTCATAGTGACC-3' \\
GAPDH (169 bp) & forward, 5'-TCACCACCATGGAGAAGGC-3' \\
& reverse, 5'-GCTAAGCAGTTGGTGGTGCA-3' \\
\hline
\end{tabular}

the same way. In the Sham group, no special treatment was performed post-laminectomy.

\section{Macroscopic assessment of EF}

Macroscopic assessment was performed four weeks post-laminectomy. Five rats were randomly selected from each group and anesthetized. Then, the surgical sites were reopened. The epidural adhesion was evaluated by assistants in a double-blinded manner based on the Rydell classification [4] (grade $0=$ epidural scar tissue was not adherent to the dura mater; grade $1=$ epidural scar tissue was adherent to the dura mater, but easily dissected; grade 2 = epidural scar tissue was adherent to the dura mater, and it was difficult to dissect without disrupting the dura matter; grade $3=$ epidural scar tissue was firmly adherent to the dura mater and could not be dissected).

\section{Determination of Hydroxyproline content (HPC) analysis} HPC analysis was performed according to a previous studies four weeks post-operatively [6,7]. Six rats in each group were selected. The scar tissue, approximately 5 mg wet weight, was collected around the laminectomy site. The samples were rinsed, homogenized, centrifuged, and hydrolyzed. One milliliter of hydroxyproline developer ( $\beta$-dimethylaminobenzaldehyde solution) was added to the samples and the standards. The absorbance at $550 \mathrm{~nm}$ was read with a spectrophotometer. HPC per milligram of scar tissue was calculated.

\section{Histological analysis}

Histological analysis was performed four weeks postlaminectomy on five rats in each group. The whole L1L2 vertebral column, including muscles and epidural scar tissue, was harvested and fixed in $10 \%$ phosphatebuffered formaldehyde solution. Decalcification and

Table 2 Grades of epidural scar adhesion in rats, according to the Rydell standard

\begin{tabular}{lllll}
\hline Group & \multicolumn{4}{c}{ Grade } \\
\cline { 2 - 5 } & $\mathbf{0}$ & $\mathbf{1}$ & $\mathbf{2}$ & $\mathbf{3}$ \\
\hline Sham & 5 & 0 & 0 & 0 \\
Vehicle & 0 & 0 & 1 & 4 \\
Sal B & 4 & 1 & 0 & 0 \\
\hline
\end{tabular}




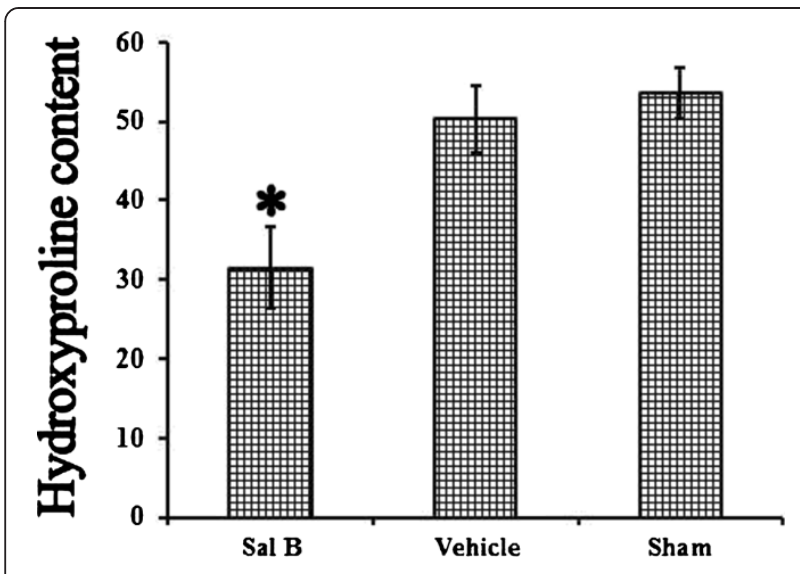

Figure 1 Hydroxyproline content was evaluated. Hydroxyproline levels are expressed as the mean \pm standard deviation of hygrotissue. The Sal B group showed the lowest hydroxyproline level. ${ }^{*} \mathrm{P}<0.05$, compared with the other two groups.

dehydration were performed with Cal-Ex II solution for 3 days. Five-micrometer axial sections of the laminectomy site were cut and stained with hematoxylin-eosin (H\&E) and Masson's trichrome.

The epidural scar adhesion was evaluated under the light microscope (Olympus ix-71). The number of fibroblasts and inflammatory cells were calculated based on a previous study [5]: grade 1, fewer than 100 fibroblasts/ inflammatory cells per $\times 400$ field; grade 2,100 to $150 \mathrm{fi}$ broblasts/inflammatory cells per $\times 400$ field; grade 3 , more than 150 fibroblasts/inflammatory cells per $\times 400$ field. Three different counting areas were selected at the middle and at the margins of the laminectomy sites. The cells were counted and their mean was calculated.

To further quantify the density of blood vessels in each scar tissue, the VEGF immunohistochemistry was performed with the monoclonal anti-VEGF antibody, and the density of VEGF was evaluated. The fields to be counted for VEGF expression were chosen at 400× magnification from well-labeled areas. Staining of VEGF was assessed with a semi-quantitative grading system that reflected the intensity of staining within 20 randomly chosen blood vessels in each scar: (0) no staining; (1) weak staining; (2) moderate staining; and (3) strong staining. Each scar was then given an overall modified $\mathrm{H}$-score ((number of vessels that scored 0$) \times 0+($ number of vessels that scored 1$) \times 1+($ number of vessels that scored 2$) \times 2+$ (number of vessels that scored 3) $\times 3$ ) $[18,19]$.

\section{Analysis of IL- 6 and TGF- $\beta 1$ concentrations}

The mRNA analyses of IL- 6 and TGF- $\beta 1$ were performed four weeks post-operatively. Six rats in each group were selected. The scar tissues were collected from the laminectomy sites, and the total RNA was extracted. The RNA $(2 \mu \mathrm{g})$ was transcribed into cDNA with AMV Reverse Transcriptase. Quantitative realtime PCR (qPCR) was performed based on a previous study using the Bio-Rad MYIQ2 (USA) [4]. Primers used are shown in Table 1. GAPDH amplification was employed as an internal control.

\section{Statistical analysis}

The statistical analysis was performed with SPSS 13.0 statistical package (SPSS Inc., Chicago, IL, USA). Data are expressed as the mean \pm standard deviation. Singlefactor analysis of variance (ANOVA) and the q-test were applied to evaluate three independent samples. Statistical significance was assumed at $\mathrm{p}<0.05$.

\section{Results}

\section{Macroscopic assessment of epidural scar adhesion}

The recovery of all rats was uneventful. All rats showed no sign of wound infection, neurological deficit or disturbance of wound healing.

In the Sal B group, soft or weak fibrous adhesion was observed. In the Vehicle and Sham groups, severe epidural adhesions were seen. The dissection of epidural scar tissue would have led to serious bleeding and the risk of dura mater disruption or nerve root injury. It was impossible to re-expose the dura mater completely. The grades of epidural scar adhesion were evaluated according to the Rydell standard (Table 2).

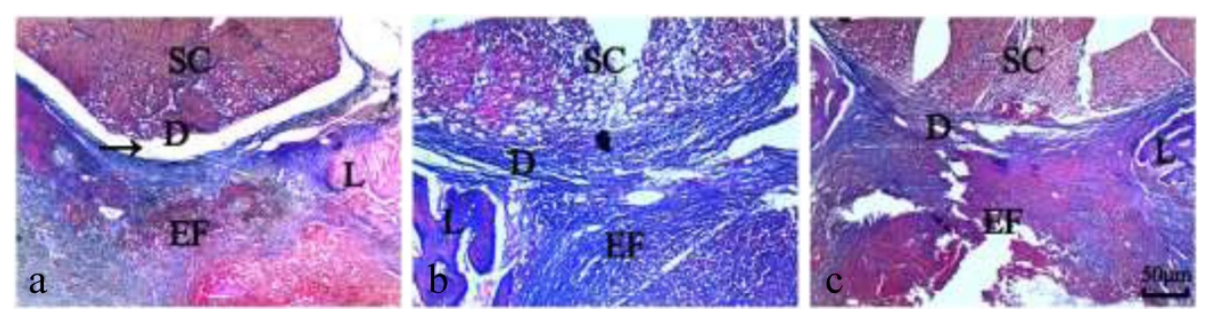

Figure 2 Masson's trichrome staining for epidural adhesion in the laminectomy sites treated with Sal B (a), Vehicle (b) or nothing (c). a: Loose scar tissues without adherence to the dura mater were seen in the Sal B group. $\mathbf{b}, \mathbf{c}$ : Dense scar tissues adhered to the dura mater were observed in the Vehicle and Sham groups. The magnification was 100x. SC = spinal cord, $D=$ dura mater, $E F=$ epidural fibrosis, $L=$ laminectomy. 

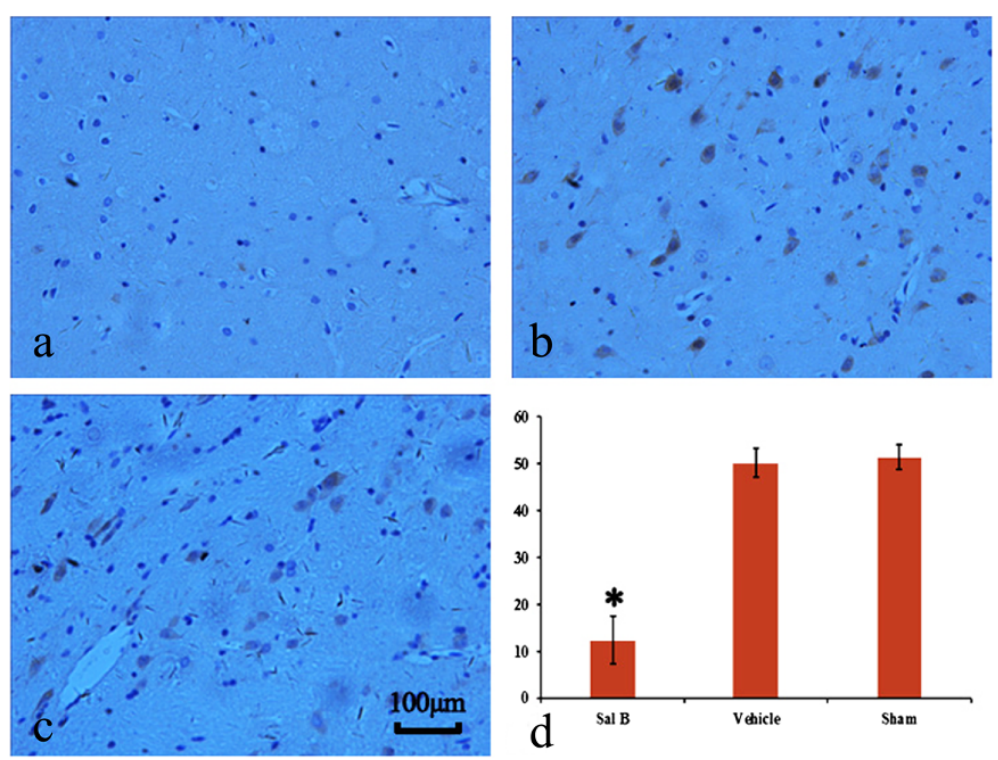

Figure 3 Effect of Sal B on vascular endothelial growth factor (VEGF). Immunohistochemistry for VEGF in epidural scar tissues treated with Sal B (a), Vehicle (b) or nothing (c). As shown in statistical analysis (d), the density of VEGF in the Sal B group (a) was less than the other 2 groups. *P $<0.05$ vs the Vehicle and the Sham group. The density of VEGF in the Vehicle group was similar to that of the Sham group. The magnification was $400 \times$.

\section{Hydroxyproline content (HPC)}

As shown in Figure 1, HPC concentration in epidural scar tissue in the Sal B group $(31.54 \pm 5.06)$ was significant lower than that in the Vehicle group $(50.34 \pm 4.19, \mathrm{P}=$ $0.006)$ and the Sham group $(53.59 \pm 3.27, \mathrm{P}=0.001)$. The content in Vehicle group showed no significant difference compared with the Sham group $(\mathrm{P}=0.249)$.

\section{Histological analysis}

In the Sal B group, loose or little epidural scar adhesion was seen (Figure 2a). In the Vehicle group and the Sham group, dense epidural scar tissue with widespread adhesions to the dura mater was observed (Figure $2 b, c)$. The immunohistochemistry analysis for VEGF showed that less VEGF was observable in the Sal B group versus the Vehicle and Sham groups. Representative sections are shown in Figure 3.

\section{Effect of Sal B on fibroblasts and inflammatory cells}

The fibroblasts and inflammatory density grades of epidural scar tissue in each group are listed in Table 3. The fibrotic and inflammatory cell densities in the Sal B group were lower than those of the Vehicle group and the Sham group. Both fibroblast and inflammatory cell densities were similar between the Vehicle group and the Sham group. Representative sections are shown in Figure 4.

Effect of Sal B on IL- 6 and TGF- $\beta 1$

The mRNA expression levels of TGF- $\beta 1$ and IL- 6 are shown in Figure 5. The Sal B group had significantly lower expression of both than the Vehicle group $(P=0.014)$ and the Sham group $(P=0.005)$. The Vehicle group and Sham group did not show a significant difference $(P=0.217)$.

\section{Discussion}

Usually, epidural fibrosis forms after laminectomy, and discectomy leads to an unfavorable clinical outcome. It

Table 3 Grades of fibroblast and inflammatory cell densities

\begin{tabular}{lll}
\hline Groups & Fibroblast density & Inflammatory cell density \\
\hline Sal B & 1 & 1 \\
Sal B 1 & 2 & 1 \\
Sal B 2 & 1 & 1 \\
Sal B 3 & 1 & 1 \\
Sal B 4 & 1 & 2 \\
Sal B 5 & & \\
Vehicle & 3 & 3 \\
Vehicle 1 & 3 & 2 \\
Vehicle 2 & 3 & 3 \\
Vehicle 3 & 3 & 3 \\
Vehicle 4 & 3 & 3 \\
Vehicle 5 & 3 & 3 \\
Sham & & 3 \\
Sham 1 & 3 & 3 \\
Sham 2 & 3 & 3 \\
Sham 3 & 3 & 3 \\
Sham 4 & 3 & \\
Sham 5 & 3 &
\end{tabular}




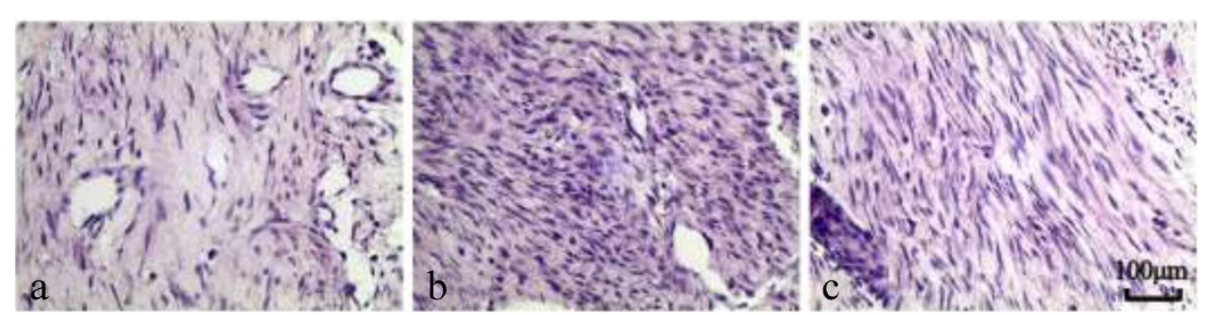

Figure 4 H\&E staining of fibroblasts in epidural scar tissues treated with Sal B (a), Vehicle (b) or nothing (c). The density of fibroblasts in the Sal B group (a) was less than those of the other 2 groups. The density of fibroblasts in the Vehicle group (b) was similar to that of the Sham group (c).

is caused by fibrous scar tissue hyperplasia, which could repair the local defect post-surgery. The fibroblast plays an important role in the repair process [7]. After being activated by inflammatory factors, it propagates and produces collagen fibers to act in a repairing role. However, local excessive inflammatory and fibrotic processes will cause excessive local collagen deposition. As a result, excessive scar tissue, transformed from fibrous connective tissue, can easily squeeze the dura mater or nerve root. Spinal stenosis, nerve root mobility restriction and dural compression could result. Therefore, it is widely accepted that $\mathrm{EF}$ is characterized by the accumulation of fibroblasts, the deposition of extracellular matrix proteins, and the distortion of normal tissue architecture with inflammation [18]. To effectively curtail EF, it is important to reduce these three factors [4].

In the present study, the potential ability of Sal B to reduce epidural scar adhesion and inhibit fibrotic and inflammatory cell proliferation at laminectomy sites was suggested. Multiple evaluations, including the Rydell classification, histological analysis, hydroxyproline content, VEGF density grade and qPCR suggested Sal B effectively prevents EF in laminectomized rats. The anti- fibrotic, anti-inflammatory, and anti-proliferative properties of Sal B have been used in different medical fields $[14,16,17]$. The present study confirms its beneficial properties in laminectomized rats. One effect of Sal B that reduced fibrosis was its downregulation of IL- 6 and TGF- $\beta 1$, which are suggested to play important roles in the promotion and/or development of EF [4-7]. Previous and current data may explain some if not all of the possible mechanisms that make Sal B effective in curtailing EF [20-22]. We hypothesize that the mechanism by which Sal B inhibits EF is its reduction of inflammatory cytokines, fibroblast proliferation and collagen deposition by blocking several signaling pathways, such as NF$\kappa B$ and ERK signaling $[16,23,24]$.

Recently, different medication have been proved to play curative effect on preventing EF. Immunosuppressive agent, antineoplastic agent and antibiotics all supported a good effectiveness on the prevention of EF [25-27]. To avoid the complication from the high-dose agents and find an optimal dose, we believe a combination between the aforementioned agent and Sal B will be a good strategy. More research will be performed. To our knowledge, this is the first study to suggest that Sal B prevents EF by

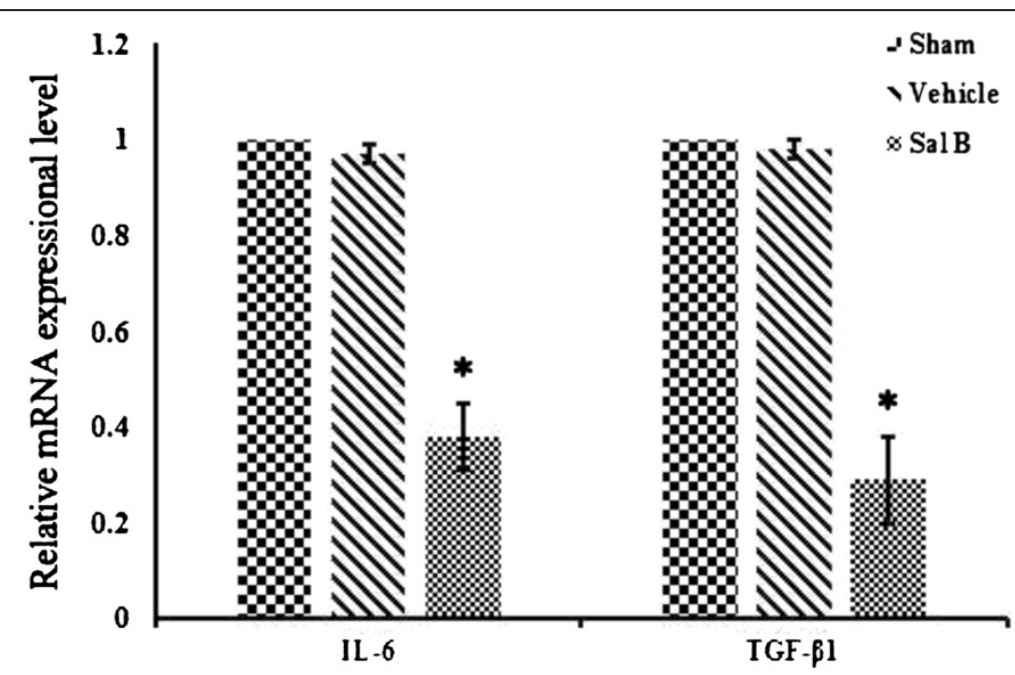

Figure 5 mRNA expression of inflammatory factors (IL-6 and TGF- $\beta 1$ ) in epidural scar tissue from each group. Data from $q P C R .{ }^{*} P<0.05$, compared with the Sham group. 
down-regulating inflammatory activity and reducing hydroxyproline deposition in rats. In the present study in EF rats, we did not attempt to define the most effective dose or high-dose toxicity. Undoubtedly, more research on the safety, long-term effects, safe effective concentration, and possible side effects and adverse effects of Sal B are all warranted before clinical trials and application.

\section{Conclusions}

Sal B can inhibit fibroblast proliferation, blood vessel regeneration, and inflammatory factor expression. Thus, Sal B is able to prevent epidural scar adhesion in postlaminectomy rats.

\section{Additional file}

\section{Additional file 1: ARRIVE guideline.}

\section{Abbreviations}

EF: Epidural fibrosis; Sal B: Salvianolic acid B; VEGF: Vascular endothelial growth factor; IL-6: Interleukin-6; TGF- $\beta 1$ : Transforming growth factor- $\beta 1$.

\section{Competing interests}

The authors declare that they have no competing interests.

\section{Authors' contributions}

FC, ZBZ and HT made substantial contributions to the conception and design, acquisition of data, analysis and interpretation of data, and drafting of the manuscript; KW performed the statistical analysis. CDZ, FGY,AYJ and HFG participated in the design and coordination of the study, and assisted with drafting the manuscript. All authors carried out the analyses, read, and approved the final manuscript.

\section{Acknowledgements}

We acknowledge all the staff in the animal experiment center of Qingdao University, who contributed great help on animal daily nursing care.

Received: 27 June 2014 Accepted: 3 October 2014

Published: 7 October 2014

\section{References}

1. Burton CV, Kirkaldy-Willis WH, Yong-Hing K, Heithoff KB: Causes of failure of surgery on the lumbar spine. Clin Orthop Relat Res 1981, 157:191-199.

2. Guyer RD, Patterson M, Ohnmeiss DD: Failed back surgery syndrome: diagnostic evaluation. J Am Acad Orthop Surg 2006, 14:534-543.

3. Siqueira EB, Kranzler LI, Dharkar DD: Fibrosis ofthe dura mater: a cause of "failed back" syndrome. Surg Neurol 1983, 19:168-170.

4. Zhang C, Kong X, Liu C, Liang Z, Zhao H, Tong W, Ning G, Shen W, Yao L, Feng S: ERK2 small interfering RNAs prevent epidural fibrosis via the efficient inhibition of collagen expression and inflammation in laminectomy rats. Biochem Biophys Res Commun 2014, 444:395-400.

5. Zhang C, Kong X, Zhou H, Liu C, Zhao X, Zhou X, Su Y, Sharma HS, Feng S: An experimental novel study: Angelica sinensis prevents epidural fibrosis in laminectomy rats via downregulation of hydroxyproline, IL-6, and TGF- $\beta$ 1. Evid Based Complement Alternat Med 2013, 2013:291814. doi:10.1155/2013/291814

6. Wang Z, Wang Y, Xie P, Liu W, Zhang S: Calcium channel blockers in reduction of epidural fibrosis and dural adhesions in laminectomy rats. Eur J Orthop Surg Traumatol 2013, [Epub ahead of print].

7. Zhang C, Kong X, Ning G, Liang Z, Qu T, Chen F, Cao D, Wang T, Sharma HS, Feng S: All-trans retinoic acid prevents epidural fibrosis through NF-KB signaling pathway in post-laminectomy rats. Neuropharmacology 2014, 79:275-281.

8. Sandoval MA, Hernandez-Vaquero D: Preventing peridural fibrosis with nonsteroidal anti-inflammatory drugs. Eur Spine J 2008, 17:451-455.
9. Henderson R, Weir B, Davis L, Mielke B, Grace M: Attempted experimental modification of the postlaminectomy membrane by local instillation of recombinant tissue-plasminogen activator gel. Spine 1993, 18:1268-1272.

10. Cokluk C, Aydi K: Experimental rabbit hemilaminotomy model in the evaluation of peridural fibrosis: a minimally invasive peridural fibrosis model. Minim Invasive Neurosurg 2005, 48:235-239.

11. Xu Q, Zhou W, Kong HY, Li L, Zhang YD, Zhang ZJ, Liu C, Wang RG: The effect of the Sanqi qisodium hyaluronate gel on the collagen of epidural scar after rabbits laminectomy. Zhongguo Gu Shang 2010, 23:278-281.

12. Joe Y, Zheng M, Kim HJ, Kim S, Uddin MJ, Park C, Ryu do G, Kang SS, Ryoo S, Ryter SW, Chang KC, Chung HT: Salvianolic acid B exerts vaso-protective effects through the modulation of heme oxygenase- 1 and arginase activities. J Pharmacol Exp Ther 2012, 341:850-858.

13. Zhou L, Zuo Z, Chow MS: Danshen: an overview of its chemistry pharmacology, pharmacokinetics, and clinical use. J Clin Pharmacol 2005 45:1345-1359.

14. Wang SX, Hu LM, Gao XM, Guo H, Fan GW: Anti-inflammatory activity of salvianolic acid $B$ in microglia contributes to its neuroprotective effect. Neurochem Res 2010, 35:1029-1037.

15. Lee YW, Kim DH, Jeon SJ, Park SJ, Kim JM, Jung JM, Lee HE, Bae SG, Oh HK, Son $\mathrm{KH}$, Ryu JH: Neuroprotective effects of salvianolic acid B on an A $325-35$ peptide-induced mouse model of Alzheimer's disease. Eur J Pharmacol 2013, 704:70-77.

16. Wang $R$, Yu XY, Guo ZY, Wang YJ, Wu Y, Yuan YF: Inhibitory effects of salvianolic acid $\mathrm{B}$ on $\mathrm{CCl}(4)$-induced hepatic fibrosis through regulating NF-kappaB/lkappaBalpha signaling. J Ethnopharmacol 2012, 144:592-598.

17. Li M, Lu Y, Hu Y, Zhai X, Xu W, Jing H, Tian X, Lin Y, Gao D, Yao J: Salvianolic acid $B$ protects against acute ethanol-induced liver injury through SIRT1-mediated deacetylation of p53 in rats. Toxicol Lett doi:10.1016/j.toxlet.2014.04.011. [Epub ahead of print].

18. Su CH, Sui T, Zhang XS, Zhang H, Cao XJ: Effect of topical application of mitomycin- $C$ on wound healing in a postlaminectomy rat model: an experimental study. Eur J Pharmacol 2012, 674:7-12.

19. Kumar I, Staton CA, Cross SS, Reed MW, Brown NJ: Angiogenesis, vascular endothelial growth factor and its receptors in human surgical wounds. Br J Surg 2009, 96:1484-1491.

20. Zhang M, Cao SR, Zhang R, Jin JL, Zhu YF: The inhibitory effect of salvianolic acid $B$ on TGF- $\beta 1$-induced proliferation and differentiation in lung fibroblasts. Exp Lung Res 2014, 40(4):172-185.

21. Stumpf C, Fan Q, Hintermann C, Raaz D, Kurfürst I, Losert S, Pflederer W, Achenbach S, Daniel WG, Garlichs CD: Anti-inflammatory effects of danshen on human vascular endothelial cells in culture. Am J Chin Med 2014, 41:1065-1077.

22. Fan ZK, LV G, Wang YF, Li G, Yu DS, Wang YS, Zhang YQ, Mei XF, Cao Y: The protective effect of salvianolic acid $B$ on blood-spinal cord barrier after compression spinal cord injury in rats. J Mol Neurosci 2013, 51:986-993.

23. Li S, Wang L, Yan X, Wang Q, Tao Y, Li J, Peng Y, Liu P, Liu C: Salvianolic acid $B$ attenuates rat hepatic fibrosis via downregulating angiotensin II signaling. Evid Based Complement Alternat Med 2012, doi:10.1155/2012/ 160726. Epub 2012 Nov 12

24. Xu D, Xu L, Zhou C, Lee WY, Wu T, Cui L, Li G: Salvianolic acid B promotes osteogenesis of human mesenchymal stem cells through activating ERK signaling pathway. Int J Biochem Cell Biol 2014, 51C:1-9.

25. Ismailoglu O, Albayrak B, Gulsen I, Tanriover G, Demir N: Topical application of tacrolimus prevents epidural fibrosis in a rat postlaminectomy model: histopathological and ultrastructural analysis. Turk Neurosurg 2011, 21:630-633.

26. Albayrak BS, Ismailoglu O, Ilbay K, Yaka U, Tanriover G, Gorgulu A, Demir N: Doxorubicin for prevention of epineurial fibrosis in a rat sciatic nerve model: outcome based on gross postsurgical, histopathological, and ultrastructural findings. J Neurosurg Spine 2010, 12:327-333.

27. Turkoglu E, Tuncer C, Dinc C, Serbes G, Oktay M, Sekerci Z: The effect of etanercept on spinal epidural fibrosis in a postlaminectomy rat model. Turk Neurosurg 2014, 24:506-511.

doi:10.1186/1471-2474-15-337

Cite this article as: Chen et al:: Study on salvianolic acid B in the reduction of epidural fibrosis in laminectomy rats. BMC Musculoskeletal Disorders 2014 15:337. 